\title{
Altered Expression of Three EGFR Posttranslational Regulators MDGI, MIG6, and EIG121 in Invasive Breast Carcinomas
}

\author{
Didier Meseure $\mathbb{D}^{1},{ }^{1,2,3}$ Kinan Drak Alsibai, ${ }^{4,5}$ Sophie Vacher, ${ }^{3}$ Rana Hatem, ${ }^{3}$ Andre Nicolas, ${ }^{1}$ \\ Celine Callens, ${ }^{3}$ Florence Lerebours, ${ }^{6}$ and Ivan Bieche ${ }^{3,7}$ \\ ${ }^{1}$ Platform of Experimental Pathology, Institut Curie, F-75248 Paris, France \\ ${ }^{2}$ Department of Diagnostic and Theranostic Medicine, Institut Curie, F-75248 Paris, France \\ ${ }^{3}$ Unit of Pharmacogenomics, Department of Genetics, Institut Curie, F-75248 Paris, France \\ ${ }^{4}$ Department of Pathology, Cayenne Hospital Center, F-97306 Cayenne, France \\ ${ }^{5}$ Center of Biological Resources (CRB Amazonie), Cayenne Hospital Center, F-97306 Cayenne, France \\ ${ }^{6}$ Department of Medical Oncology, Institut Curie, Rene Huguenin Hospital, F-92210 Saint-Cloud, France \\ ${ }^{7}$ Faculty of Pharmaceutical and Biological Sciences, Sorbonne Paris Cité, Paris Descartes University, F-75006 Paris, France
}

Correspondence should be addressed to Didier Meseure; didier.meseure@curie.fr

Received 14 June 2019; Revised 11 November 2019; Accepted 6 March 2020; Published 20 April 2020

Academic Editor: Dorota L. Stankowska

Copyright ( 2020 Didier Meseure et al. This is an open access article distributed under the Creative Commons Attribution License, which permits unrestricted use, distribution, and reproduction in any medium, provided the original work is properly cited.

\begin{abstract}
Epidermal growth factor receptor (EGFR) signalling is a highly regulated process with a tight balance between receptor activation and inactivation in invasive breast carcinomas (IBCs) particularly in triple-negative carcinomas (TNC). Clinical trials using antiEGFR therapies are actually performed although no activating alterations (mutations, amplifications, or rearrangements) of EGFR have been clearly recognized in order to identify new targeted modalities for IBCs. We explored mammary-derived growth inhibitor (MDGI), estrogen-induced gene-121 (EIG121), and mitogen-induced gene-6 (MIG6), three posttranslational EGFR trafficking molecules implicated in EGFR spatiotemporal regulatory pathway. We quantified MDGI, EIG121, and MIG6 at mRNA levels by using real-time quantitative RT-PCR in a series of 440 IBCs and at protein levels by using immunohistochemistry in a series of 88 IBCs. Results obtained by RT-PCR showed that in IBCs, MDGI, MIG6, and EIG121 mRNA were mainly underexpressed $(25.7 \%, 45.0 \%$, and $16.1 \%$, respectively) particularly in the TNC subtype for EIG121 (60.3\%). We also observed mRNA overexpression of MDGI and EIG121, respectively, in $12.7 \%$ and $22.3 \%$ of IBCs. These altered mRNA expressions were confirmed at the protein level. Some links were found between expression patterns of these three genes and several classical pathological and clinical parameters. Only EIG121 was found to have a prognostic significance $(p=0.0038)$. Altered expression of these three major EGFR posttranslational negative regulators could create an aberrant EGFR-mediated oncogenic signalling pathway in IBCs. MDGI, MIG6, and EIG121 expression status also may be potential useful biomarkers (sensitivity or resistance) in targeted EGFR therapy.
\end{abstract}

\section{Introduction}

Epidermal growth factor receptor (EGFR) is the founding member of the ErbB receptor tyrosine kinase (RTK) family. RTKs, which contain an extracellular ligand binding domain, a transmembrane domain, and an intracellular tyrosine kinase domain, mediate cellular signal transduction by extracellular ligand binding. The EGFR family of RTKs consists of four members: EGFR/ErbB-1/HER-1, ErbB-2/HER-2/neu, ErbB3/HER-3, and ErbB-4/HER-4 [1-3]. Upon ligand binding,
EGFR family proteins dimerize via receptor homodimerization or heterodimerization and subsequently induce tyrosine kinase activity. Activated EGFR family receptors trigger numerous downstream signalling pathways, such as phosphatidylinositol-3 kinase (PI3K), mitogen-activated protein kinase (MAPK), signal transducer and activator of transcription (STAT), and phospholipase C (PLC), and modulation of calcium channels. These downstream signalling activities control proliferation, mobility and differentiation in morphogenesis, homeostasis, and wound healing. The 
crucial role of EGFR in these physiological events is evidenced by the embryonic lethality of EGFR knockout animals and tissue defects in EGFR ligand knockout animals $[4,5]$. Many carcinomas, including those affecting the lung, colon, and kidney, are characterized by overexpression and/or gene alteration that activate EGFR [6-8]. EGFR signalling activation has been linked with resistance to cytotoxic drugs, hormone, and anti-EGFR therapies and is an indicator of poor prognosis. In IBCs and particularly the TNC subtype, clinical trials using anti-EGFR therapies are actually performed although no activating alterations, including EGFR mutations, amplifications, or rearrangements have been clearly identified. Moreover, EGFR expression levels in IBCs have not been shown to correlate with cancer responsiveness and recent data have suggested that EGFR mRNA is not frequently overexpressed as previously reported but surprisingly mainly underexpressed compared with normal tissues [9]. Therefore, a complete understanding of EGFR functions has important implications in cancer biology. Furthermore, the identification of regulatory mechanisms and molecular basis of sensitivity/resistance to EGFR inhibitors will help to establish a rational basis for targeted therapies.

EGFR signalling is a highly regulated process with a tight balance between activation and inactivation of the receptor. However, this process is much more complex than it first seemed due to various mechanisms recently identified that regulate EGFR signalling. Numerous molecular mechanisms classically impact EGFR signalling, including ligand concentration, receptor density, duration of activated receptors, and proximity of EGFR to downstream effectors. Among them, the endocytic pathway has recently emerged as a key spatiotemporal regulator of EGFR [10]. To reduce the level of a specific plasma membrane protein in a short period, cells internalize the protein from the cell surface by endocytosis and degrade it in the lysosomal compartment. It is now widely accepted that "endocytic matrix" is a master organizer of signalling, governing resolution of signals in space and time. Consequently, endocytosis affects crucial cell functions that range from proliferation to cell motility. Recent data suggest that cancer could be related to alteration of subcellular protein localization, trafficking, and compartmentalization [11]. At least three mechanisms have been proposed to explain how endocytic trafficking pathway deregulation could contribute to malignant transformation, including receptor dephosphorylation, removing receptor from cell surface, and targeting ligand-receptor complex for degradation. To explore the endocytic pathway in IBCs, we analyzed three major EGFR trafficking molecules implicated in EGFR sequestration (mammary-derived growth inhibitor (MDGI) also called FABP3), EGFR endolysosomal degradation (estrogen-induced gene-121 (EIG121) also called KIAA1324), and EGFR inhibition/dephosphorylation (mitogen-induced gene6 (MIG6) also called ERRFI1).

The aim of this study was to identify new targeted modalities for IBC treatments by quantifying MDGI, EIG121, and MIG6 mRNA levels in a series of 440 IBCs from patients with known clinical and pathological status and long-term outcome. We also analyzed MDGI, EIG121, and MIG6 at protein levels in a series of 88 IBCs.

\section{Materials and Methods}

2.1. Patients and Samples. We obtained tumor samples from 440 patients treated at Curie Institute, Rene Huguenin Hospital, (Saint-Cloud, France) from 1978 to 2008. All patients who entered our institution before 2007 were informed that their tumor samples might be used for scientific purposes and had the opportunity to decline. Since 2007, patients entering our institution have given their approval also by signed informed consent. Tumor samples containing more than $70 \%$ of tumor cells were considered suitable for analysis. Immediately after surgery, the tumor samples were placed in liquid nitrogen until RNA extraction. The patients met the following criteria: primary unilateral nonmetastatic breast carcinoma; complete clinical, histological, and biological information available; no preoperative radiotherapy or chemotherapy; and complete follow-up at our institute. Patients underwent physical examination every 3 months for 2 years, then annually. Mammograms were performed annually. Median follow-up was 8.9 years (range 6 months to 29 years).

Ten specimens of adjacent normal breast tissue from breast cancer patients on normal breast tissue from women undergoing cosmetic breast surgery were used as sources of normal RNA.

The histological type and the number of positive axillary nodes were established at the time of surgery. IBCs were scored according to Scarff, Bloom, and Richardson (SBR) histopronostic system. Hormone receptor (HR) estrogen and progesterone receptors (ER and $\mathrm{PR}$ ) were routinely analyzed at the time of diagnosis on frozen tumors using ligand binding assay until 1988, enzyme immunoassay (ER-EIA Monoclonal, PgR-EIA Monoclonal, Abbott Laboratories, Abbott Park, IL) between 1988 and 2000, and then immunohistochemistry on paraffin sections. Tyrosine kinase receptor HER2 status was routinely analyzed by immunohistochemistry (with confirmation by FISH of the $2+$ cases). In this study, the tumor subtype status was confirmed by RT-PCR on frozen samples [12,13]. According to HR (ER and PR) and HER2 status, the total population $(n=440)$ was subdivided in 4 subtypes: subtype $1(\mathrm{HR}+(\mathrm{ER}+$ or/and $\mathrm{PR}+)$ and HER2 +$)(n=50)$; subtype $2(\mathrm{HR}+(\mathrm{ER}+$ and/or $\mathrm{PR}+)$ and HER-) $(n=281)$; subtype 3 (HR- (ER- and PR-) and HER2+) $(n=46)$; and subtype 4 that corresponds to the TNC subtype (ER-, PR- and HER2-) $(n=63)$.

2.2. RNA Extraction. Total RNA was extracted from breast tissue samples by using acid-phenol guanidinium as previously described [14]. RNA quality was determined by electrophoresis through agarose gels, staining with ethidium bromide, and visualization of the $18 \mathrm{~S}$ and $28 \mathrm{~S}$ RNA bands under ultraviolet light.

2.3. Real-Time RT-PCR. Quantitative values were obtained from the cycle number (Ct value) at which the increase in the fluorescence signal associated with exponential growth of PCR products started to be detected by the laser detector of the ABI Prism 7900 sequence detection system (PerkinElmer Applied Biosystems, Foster City, CA), using PE Biosystems analysis software according to the manufacturer's 
manuals. The precise amount of total RNA added to each reaction mix (based on optical density) and its quality (i.e., lack of extensive degradation) are both difficult to assess. Therefore, transcripts of the TBP gene (GenBank accession NM_003194) encoding the TATA box-binding protein (a component of the DNA-binding protein complex TFIID) were also quantified as an endogenous RNA control. Each sample was normalized on the basis of its TBP content. $T B P$ was selected as an endogenous control due to the moderate prevalence of its transcripts and the absence of known TBP retropseudogenes (retropseudogenes lead to coamplification of contaminating genomic DNA and thus interfere with RT-PCR, despite the use of primers in separate exons) [13]. Results, expressed as $N$-fold differences in target gene expression relative to the TBP gene and termed "Ntarget," were determined as Ntarget $=2^{\Delta \text { Ctsample }}$, where the $\Delta \mathrm{Ct}$ value of the sample was determined by subtracting the average $\mathrm{Ct}$ value of the target gene from the average $\mathrm{Ct}$ value of the $T B P$ gene. In these mRNA and protein series, we have previously analyzed EGFR expression [9]. MDGI, EIG121, and MIG6 mRNA expressions were normalized to the housekeeping gene TBP (which encodes the TATA box-binding protein), and the median relative expression level in the 10 normal breast tissues was attributed a value of 1. MDGI, EIG121, and MIG6 mRNA expression levels in the 10 normal breast samples were 0.6 to 1.6. The threshold value of 2 was then considered overexpression and $\leq 0.5$ underexpression in breast cancer tissues, as reported in previous studies [9, $15,16]$. The conditions of cDNA synthesis and PCR were as previously described [13].

2.4. Immunohistochemistry. Formalin-fixed and paraffinembedded tissue blocks, obtained at the time of initial diagnosis, were retrieved from of the Department of Pathology archives. Sections of $3 \mu \mathrm{m}$ in thickness were cut with a microtome from the paraffin-embedded tissue blocks of normal breast tissue and IBCs. Tissue sections were deparaffinised and rehydrated through a series of xylene and ethanol washes. Immunohistochemical assays were performed in the series of 88 IBCs: subtype $1(\mathrm{HR}+(\mathrm{ER}+$ or/and $\mathrm{PR}+)$ and HER2 +$)(n=13)$; subtype $2(\mathrm{HR}+(\mathrm{ER}+$ and/or $\mathrm{PR}+)$ and HER-) $(n=47)$; subtype 3 (HR- (ER- and PR-) and HER2 +$)(n=10)$; and subtype 4 (ER-, PR- and HER2-) $(n=18)$ using a panel of antibodies against EIG121 (polyclonal anti-EIG121, dilution 1/300, Novus Biologicals ${ }^{\mathrm{TM}}$ ); MDGI (polyclonal anti-MDGI, dilution 1/50, Spring Bioscience $^{\mathrm{TM}}$ ); and MIG6 (polyclonal anti-ERRFI1, dilution 1/50, Proteintech $^{\mathrm{TM}}$ ). Positive IHC reactions were defined as a cytoplasmic staining for EIG121, MDGI, and MIG6.

According to manufactory datasheet, positive immunohistochemistry control was performed using normal endometrial tissue, colon adenocarcinoma, and tonsillitis tissue for EIG121, MDGI, and MIG6, respectively. In breast tissue, we used terminal ductulolobular units (TDLU) as internal positive control, and we verified that antigen retrieval was utilized correctly, all reagents were mixed and applied properly, antibody dilutions and the method of staining were correct, and the incubation times and temperatures were optimal. We also performed both negative reagent and inter- nal negative controls to ensure specificity and sensitivity of the three antibodies and rule out additional or nonspecific staining when the primary antibody is removed from the protocol.

In our series, a protein IHC intensity score (IHC score 0 to 3) was attributed by comparing neoplastic cells to normal epithelial cells of TDLU.

2.5. Statistical Analysis. The distributions of target (mRNA and protein) expression levels were characterized by their median values and ranges. Relationships between mRNA levels of the different target genes, and between mRNA levels and clinical parameters, were identified by using nonparametric tests, namely, the chi-square test (relation between 2 qualitative parameters), and the Spearman rank correlation test (relation between 2 quantitative parameters). Differences were considered significant at confidence levels greater than 95\% $(p<0.05)$.

Metastasis-free survival (MFS) was determined as the interval between initial diagnosis and detection of the first metastasis. Survival distributions were estimated by the Kaplan-Meier method, and the significance of differences between survival rates was ascertained with the log-rank test.

\section{Results}

Results obtained by RT-PCR (Figure 1) showed that MIG6 was mainly underexpressed in our breast tumor series (underexpression $45.0 \%$, overexpression 5.5\%; median mRNA level, 0.53 ; range, 0.00-5.93) and more particularly in the $\mathrm{HR}+1$ HER2- subtype (51.2\%). Both overexpression and underexpression were observed for the two other genes: MDGI (underexpression 25.7\%, overexpression 12.7\%; median mRNA level, 0.76; range, 0.14-26.84), and EIG121 (underexpression $16.1 \%$, overexpression $22.3 \%$; median mRNA level, 1.19; range, 0.00-15.73). MDGI and EIG121 were mainly overexpressed in the two HR+ subtypes. TNCs showed high frequency of EIG121 underexpression (60.3\%). Table 1 also shows mRNA levels of EGFR previously analyzed in the same series of 440 IBCs [9]. EGFR mRNA was underexpressed in IBCs relative to normal breast tissues (HR+/HER2+: 88.0\%; HR+/HER2-: 91.5\%; HR-/HER2+: 69.6\%; and HR-/HER2-: $63.5 \%)$. EGFR mRNA was overexpressed in only $6.3 \%$ of triple-negative (HR-/HER2-) tumors and in almost none of the tumors in the other 3 subtypes (Table 1).

MDGI, MIG6, and EIG121 altered expressions were confirmed at the protein level by using IHC in the total population of IBCs tested $(n=88)$ and in the different subtypes. In our series of 88 IBCs, protein underexpression (IHC score 0 ) of the 3 inhibitors was identified in $19.4 \%$ (MDGI), 43.2\% (MIG6), and 19.4\% (EIG121). Interestingly, we could confirm that EIG121 underexpression was observed in $61.1 \%$ of IBCs of the TNC subtype and MIG6 underexpression in $55.3 \%$ of HR+/HER2- IBCs (Table 2 and Figures 2 and 3). These similar results obtained both at the mRNA and protein levels suggest that regulation of these 3 genes is mainly transcriptional.

To test the relationship between EGFR, MDGI, MIG6, and EIG121 gene mRNA levels in the series of 440 IBCs, we 

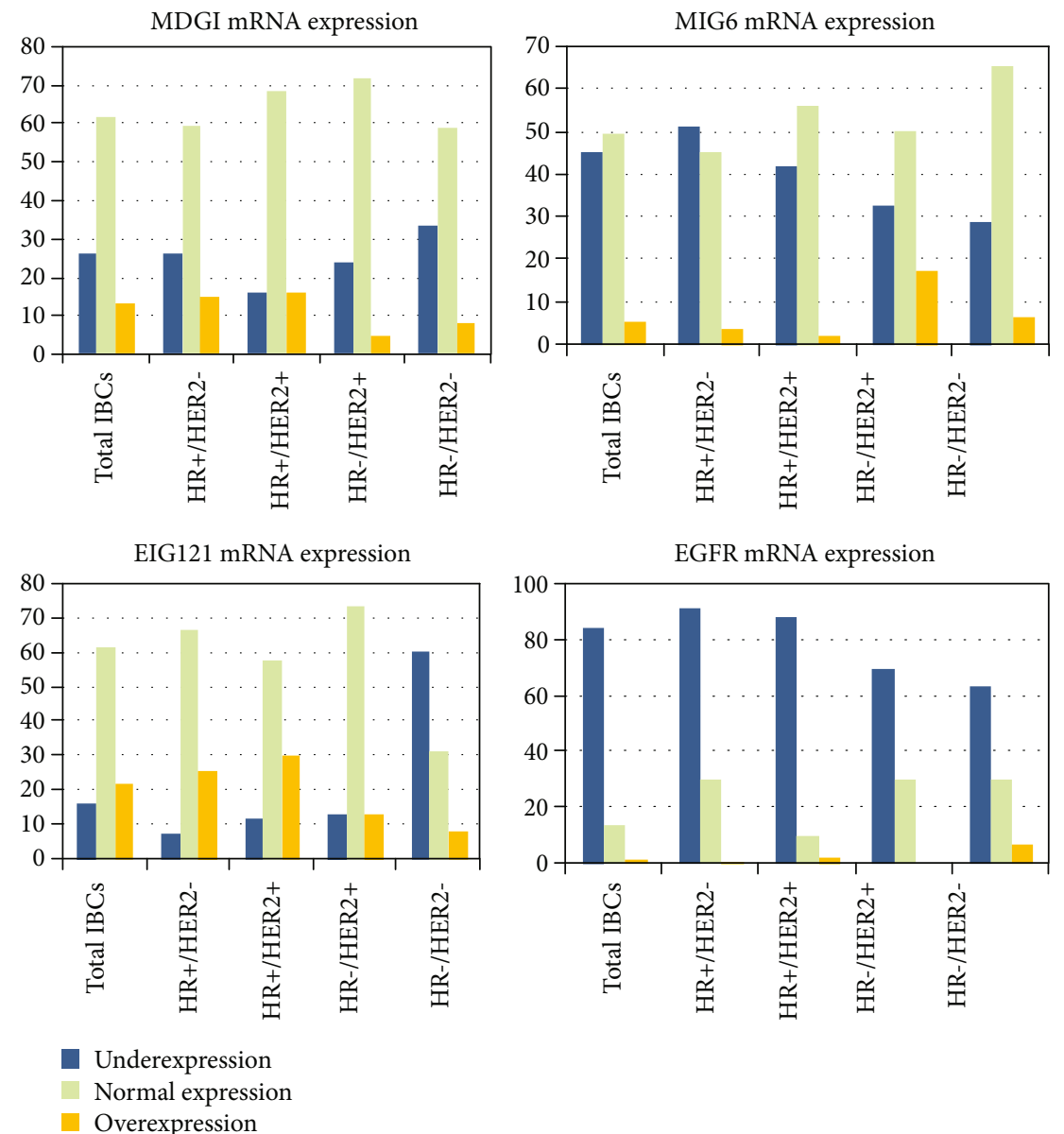

FIGURE 1: Results obtained by RT-PCR showed that MIG6 is mainly underexpressed in invasive breast carcinomas and more particularly in the HR+/HER2- subtype. Both overexpression and underexpression were observed for the two other genes: MDGI and EIG121 are mainly overexpressed in the two HR+ subtypes. TNCs showed high frequency of EIG121 underexpression.

TABLE 1: MDGI, MIG6, EIG121, and EGFR mRNA expression by RT-PCR in breast tumors $(n=440)$.

\begin{tabular}{|c|c|c|c|c|c|c|c|}
\hline Genes & mRNA & $\begin{array}{l}\text { All tumors } \\
n=440 \\
<0.5 N>2\end{array}$ & $\begin{array}{c}\text { HR-/HER2- }(\mathrm{TNC}) \\
n=63 \\
<0.5 \mathrm{~N}>2\end{array}$ & $\begin{array}{c}\text { HR-/HER2+ } \\
n=46 \\
<0.5 N>2\end{array}$ & $\begin{array}{c}\text { HR+/HER2- } \\
n=281 \\
<0.5 N>2\end{array}$ & $\begin{array}{c}\mathrm{HR}+/ \mathrm{HER} 2+ \\
n=50 \\
<0.5 \mathrm{~N}>2\end{array}$ & $p$ value* \\
\hline \multirow{2}{*}{$M D G I$} & 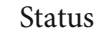 & $25.7 \%$ 61.6\% $12.7 \%$ & $33.3 \% 58.8 \% 7.9 \%$ & $23.9 \% 71.8 \% 4.3 \%$ & $26.0 \% 59.4 \% 14.6 \%$ & $16.0 \% 68.0 \% 16.0 \%$ & \multirow{2}{*}{0.14 (NS } \\
\hline & Level & $0.76(0.14-26.84)$ & $0.62(0.15-26.84)$ & $0.83(0.20-4.78)$ & $0.75(0.14-18.16)$ & $0.96(0.29-7.73)$ & \\
\hline \multirow{2}{*}{ MIG6 } & atus & $45.0 \%$ 49.5\% 5.5\% & $28.6 \% 65.1 \% 6.3 \%$ & $32.6 \% 50.0 \% 17.4 \%$ & $51.2 \%$ 44.9\% 3.9\% & $42.0 \% 56 \% 2.0 \%$ & \multirow{2}{*}{0.0001} \\
\hline & Level & $0.53(0.00-5.93)$ & $0.71(0.08-3.96)$ & $0.66(0.13-5.93)$ & $0.49(0.00-5.34)$ & $0.54(0.00-3.86)$ & \\
\hline \multirow{2}{*}{ EIG121 } & atus & $16.1 \%$ 61.6\% 22.3\% & $60.3 \% 31.8 \% 7.9 \%$ & $13.0 \% 74.0 \% 13.0 \%$ & $7.5 \%$ 66.9\% 25.6\% & $12.0 \% 58.0 \% 30.0 \%$ & \multirow{2}{*}{$<0.0001$} \\
\hline & Level & $1.19(0.00-15.73)$ & $0.33(0.00-4.53)$ & $0.93(0.21-4.41)$ & $1.36(0.08-15.73)$ & $1.49(0.23-6.65)$ & \\
\hline \multirow{2}{*}{$E G F R$} & Status & $84.8 \% 13.8 \% 1.4 \%$ & $63.5 \% 30.2 \% 6.3 \%$ & $69.6 \% 30.4 \% 0.0 \%$ & $91.5 \% 8.1 \% 0.4 \%$ & $88 \% 10.0 \% 2.0 \%$ & \\
\hline & Level & $0.16(0.01-82.84)$ & $0.44(0.03-82.84)$ & $0.20(0.04-1.67)$ & $0.13(0.01-7.30)$ & $0.15(0.03-2.58)$ & \\
\hline
\end{tabular}

${ }^{*}$ Chi-squared test: total $p$ value $(n=440)$.

used the Spearman rank correlation test for continuous variables (Supplementary Table 1S) and only found a weak positive correlation $(r=+0.094, p=0.045)$ between EGFR and MDGI mRNA levels, reflecting very independent interplay across this four-gene set.
We sought links between MDGI, MIG6, and EIG121 qualitative mRNA status and standard clinical, pathological, and biological factors. In IBCs, determination of PIK3CA status is important for diagnosis, prognosis, prediction of resistance, and theranostics. PIK3CA mutations are particularly 
TABLE 2: MDGI, MIG6, EIG121, and EGFR protein expression by IHC in breast tumors $(n=88)$.

\begin{tabular}{lcccccc}
\hline & All tumors & HR-/HER2- (TNC) & HR-/HER2+ & HR+/HER2- & HR+/HER2+ \\
Protein & $n=88$ & $n=18$ & $n=10$ & $n=47$ & 013 \\
& $012 / 3$ & $012 / 3$ & $012 / 3$ & $012 / 3$ & $012 / 3$ \\
\hline MDGI & $19.4 \% 67 \% 13.6 \%$ & $27.7 \% 66.6 \% 5.7 \%$ & $10 \% 70 \% 20 \%$ & $19.1 \% 68 \% 12.9 \%$ & $15.3 \% 61.7 \% 23 \%$ \\
MIG6 & $43.2 \% 46.6 \% 10.2 \%$ & $22.2 \% 61.1 \% 16.7 \%$ & $30 \% 60 \% 10 \%$ & $55.3 \% 38.2 \% 6.5 \%$ & $38.4 \% 46.3 \% 15.3 \%$ & $0.27(\mathrm{NS})$ \\
EIG121 & $19.4 \% 57.9 \% 22.7 \%$ & $61.1 \% 33.3 \% 5.6 \%$ & $20 \% 70 \% 10 \%$ & $6.3 \% 63.8 \% 29.9 \%$ & $7 \% 61.5 \% 31.5 \%$ & $<0.0001$ \\
EGFR & $59.1 \% 26.1 \% 14.8 \%$ & $27.7 \% 0 \% 72.3 \%$ & $40 \% 60 \% 0 \%$ & $76.5 \% 23.5 \% 0 \%$ & $53.0 \% 47.0 \% 0 \%$ \\
\hline
\end{tabular}

${ }^{*}$ Chi squared test: total $p$ value $(n=88)$. IHC score 0 : underexpression; score 1: normal expression; score 2/3: overexpression.
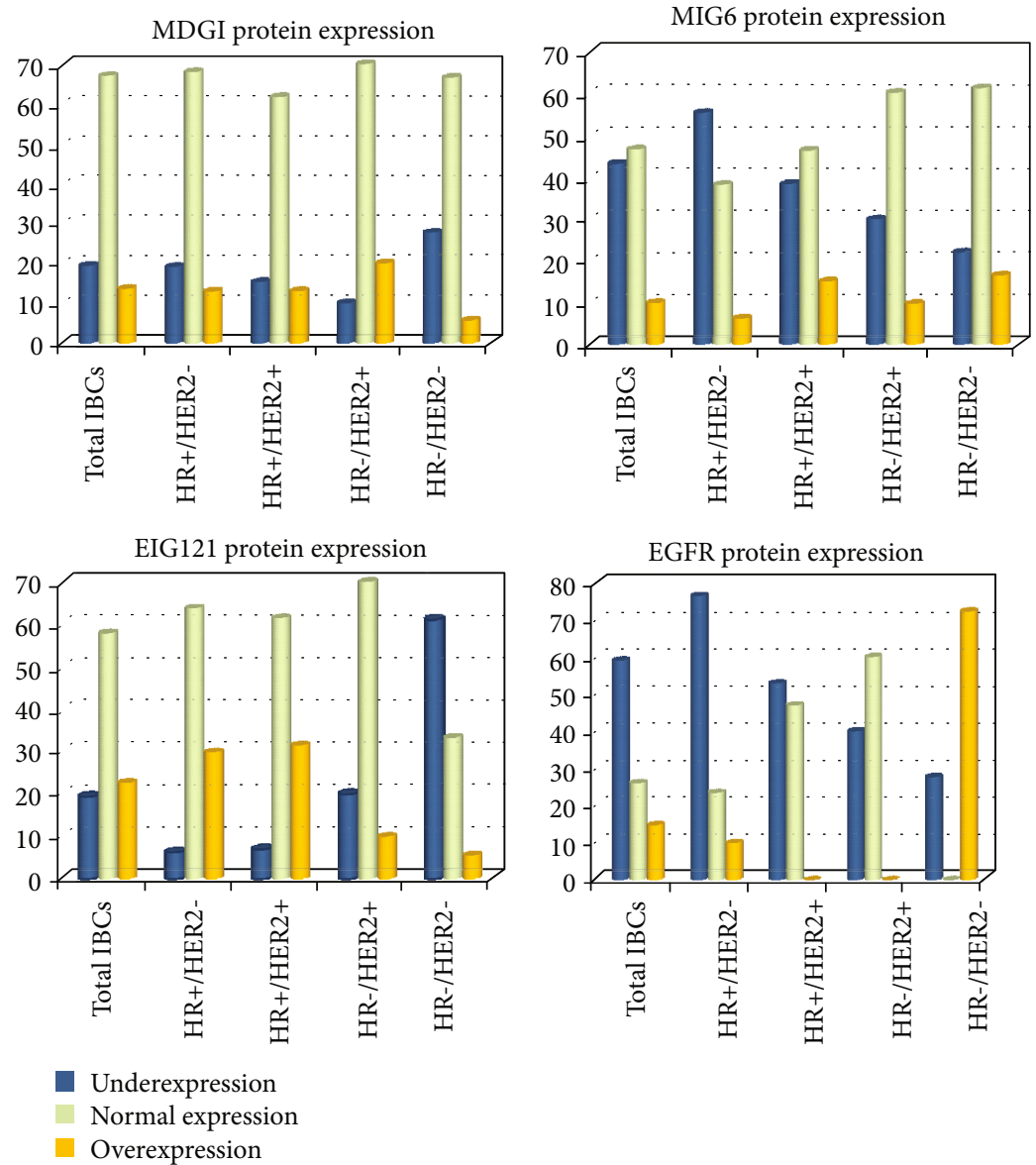

FIGURE 2: Results of MDGI, MIG6, and EIG121 confirmed at a protein level by using IHC in a population of invasive breast carcinomas $(n=88)$. Protein underexpression of the 3 inhibitors is identified in 19.4\% (MDGI), 43.2\% (MIG6) and 19.4\% (EIG121). Interestingly, EIG121 underexpression is observed in $61.1 \%$ of the TNC subtype and MIG6 underexpression in 55.3\% of the HR+/HER2- subtype.

frequent in $\mathrm{HR}+$ breast carcinomas and potent drivers of carcinogenesis through AKT activation, evasion of apoptosis, and promotion of invasion. PIK3CA somatic mutations are correlated with significantly better clinical outcome in early-stage IBCs and are associated with resistance to paclitaxel, trastuzumab, and endocrine treatment [17]. We observed in our series significant positive correlation between EIG121 transcripts and PIK3CA mutation status ( $p=$ 0.00067) (Supplementary Tables 2S-4S, respectively). MDGI mRNA level was highly associated with lymph node status (Supplementary Table 2S). MIG6 mRNA level was markedly associated with lymph node status, ER status, and molecular subtypes (Supplementary Table 3S). EIG121
mRNA level was markedly associated with SBR histological grade, lymph node status, ER and PR status, molecular subtypes, and PIK3CA mutation status (Supplementary Table 4S). One simple explanation to the observed positive association between high mRNA level of EIG121 and PIK3CA mutation is that high mRNA level of EIG121 is also associated with estrogen receptor-positive tumors which are known to have a high proportion of PIK3CA mutations [16].

Finally, we used a log-rank test to identify relations between metastasis-free survival (MFS) and MDGI, MIG6, and EIG121 gene mRNA levels. The difference in MFS among patients with downregulated, normal, and upregulated EIG121 expression was statistically significant $(p=0.0038)$ (Figure 4). 

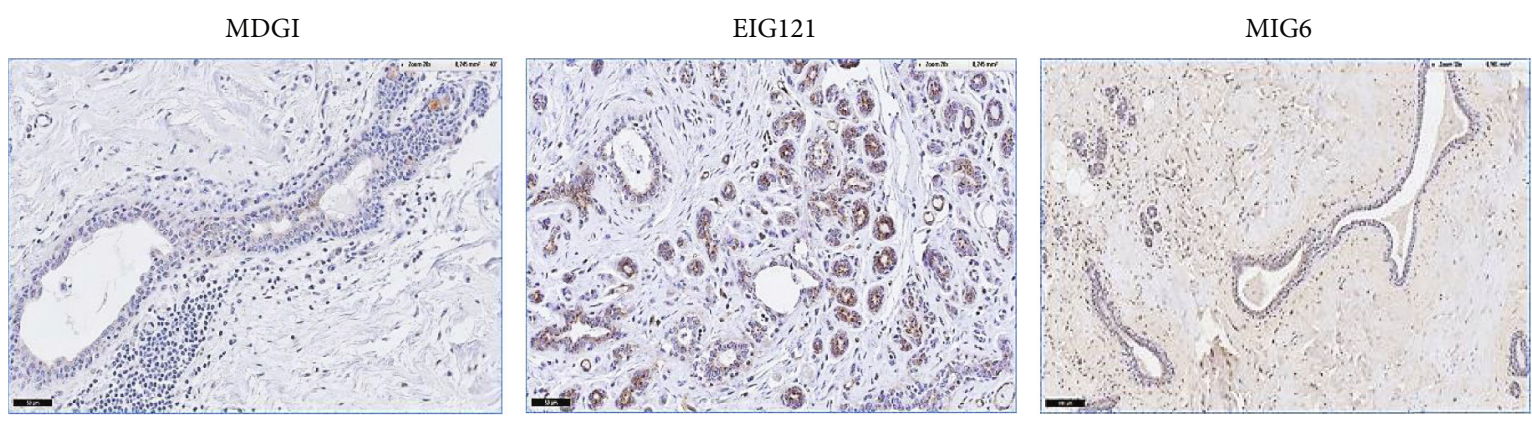

(a)
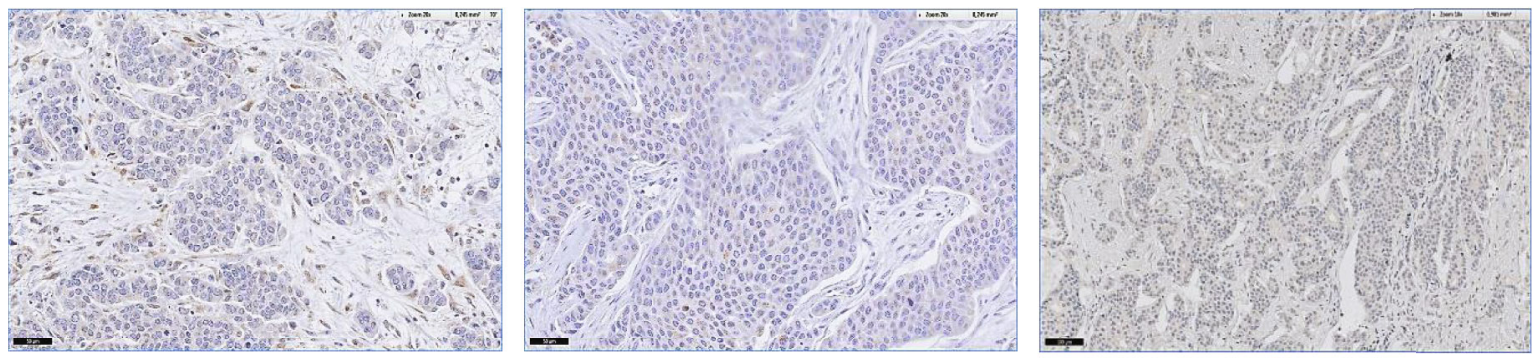

(b)
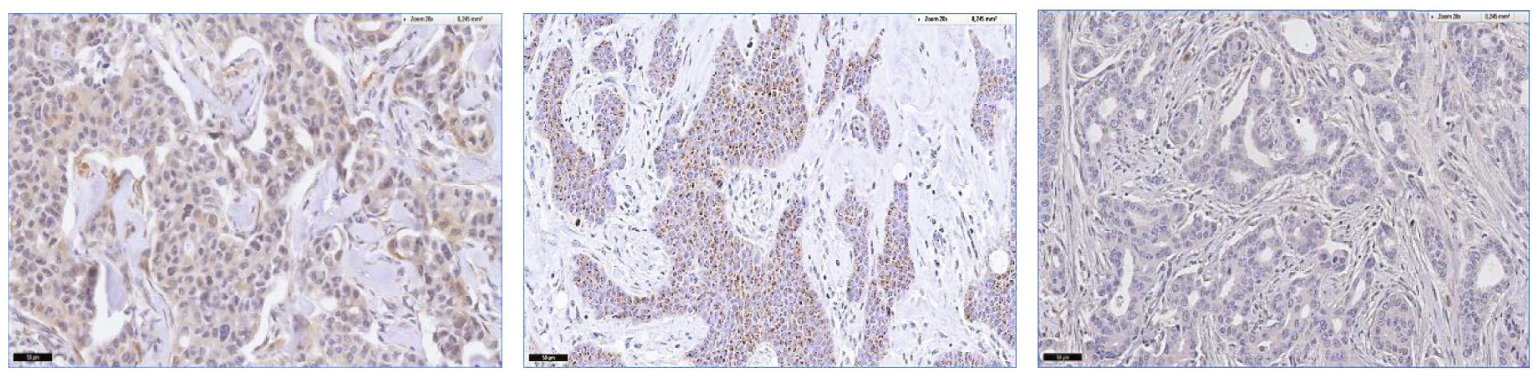

(c)
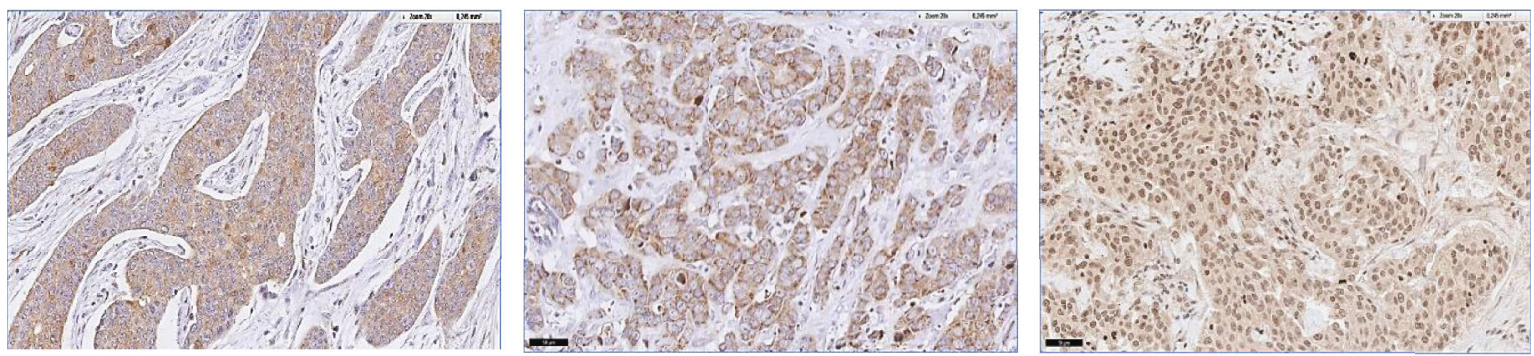

(d)
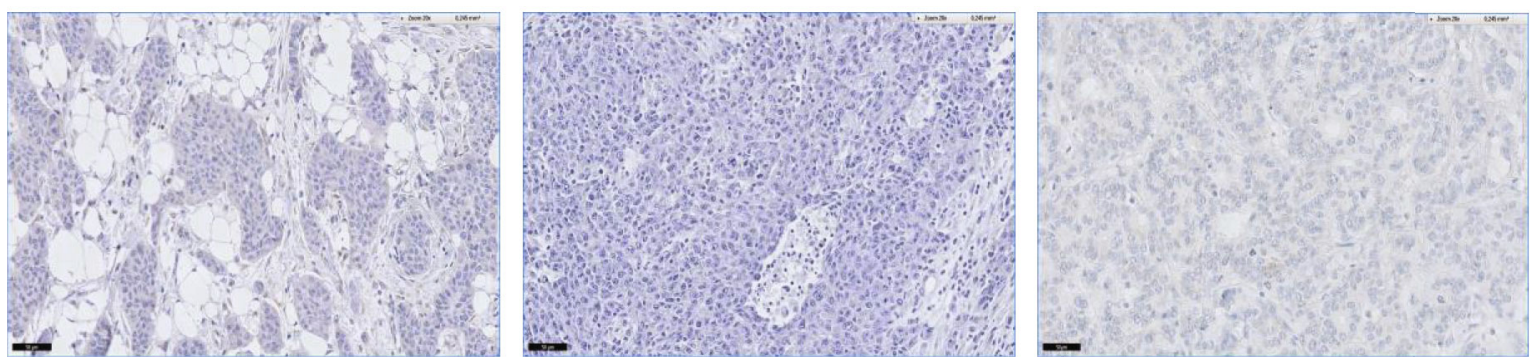

(e)

Figure 3: MDGI, EIG121, and MIG6 immunohistochemical score (IHC) in terminal ductulolobular units (TDLU) and invasive breast carcinomas (IBC). (a) Normal TDLU; (b) IBC: IHC score 1; (c) IBC: IHC score 2; (d) IBC: IHC score 3; (e) IBC: IHC score 0. 


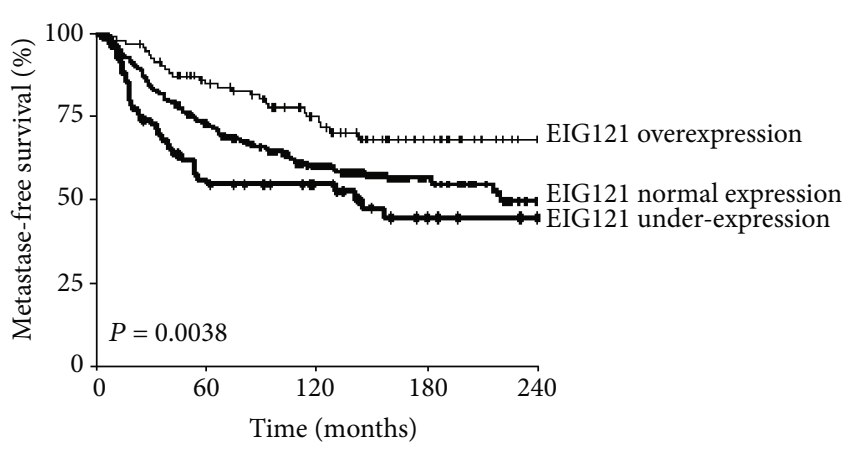

Figure 4: Relations between metastasis-free survival (MFS) and MDGI, MIG6, and EIG121 gene mRNA levels. The difference in MFS among patients with downregulated, normal, and upregulated EIG121 expression was statistically significant $(p=0.0038)$.

Patients with the poorest prognosis showed EIG121 underexpression (5-year RFS 56.9\% (50.9-62.9); 15-year RFS 45.3\% (38.6-52.1)), while those with the best prognosis had EIG121 overexpression (5-year RFS 85.4\% (81.8-89.0); 15year MFS 68.8\% (63.5-74.9)). The third subgroup with normal EIG121 expression had an intermediate outcome (5-year MFS 73.4\% (70.7-76.1); 15-year MFS 57.1\% (53.8-60.5)). MFS was not influenced by expression status of the two other genes, MDGI and MIG6.

\section{Discussion}

For over 25 years, the field has evolved from viewing endocytosis as strictly a negative regulator of the ligand receptor/complex to now appreciating its complexity in both positively and negatively modulating receptor-effector communications [11]. In addition to initiating activation of downstream signalling pathways, EGF binding also causes ligand-receptor complex to internalize via clathrin or less frequently caveolae or various small molecular weight GTPbinding proteins (Arf6, RhoA, and Cdc42). Liganded EGFR monomers dimerize and translocate along the plasma membrane until association with a membrane domain that is enriched with clathrin on the intracellular face. This domain invaginates to initiate a clathrin-coated pit, which pinches off to form a clathrin-coated vesicle. Clathrin is shed from this primary vesicle to produce an intermediate vesicle that fuses with and delivers EGF/EGFR complex to an early endosome (Figure 5). Ligand/receptor complex is then readied for its ultimate cellular fate: (i) the early endosome matures into a late endosome and delivers cargo to a lysosome, (ii) recycling endosome pinches off the early endosome and ligand and receptor recycle back to plasma membrane, or (iii) an endosome forms to deliver receptor to some other intracellular organelles (mitochondria and nucleus via the trans-Golgi network and endoplasmic reticulum) [18].

Each endocytic route has a very different consequence on EGFR signalling. Traffic to lysosomes results in attenuated signalling, due to receptor degradation. Recycling back to the plasma membrane allows EGFR restimulation by extracellular ligands. Transporting via the Golgi apparatus and endoplasmic reticulum to the nucleus allows EGFR to act as a transcriptional regulator involved in cell proliferation, tumor progression, DNA repair, and chemo- and radioresistance [19]. Moreover, recent data have demonstrated that the endocytic pathway is critical for appropriate spatial localization of ligand-receptor complex to activate downstream effectors. Inhibition of EGFR endocytosis decreases efficiency of signalling to MAPK and PI3K and induction of apoptosis. Further, maintaining active EGFR at the plasma membrane enhances phosphorylation of EGFR and DNA synthesis. Thus, this trafficking endomembrane system determines the strength and duration of signalling responses not only by controlling sorting events such as recycling and transporting to a lysosomal compartment for ligand/receptor degradation but also by recruiting downstream effectors of signalling complexes.

We previously showed that EGFR was markedly underexpressed both at the mRNA and protein levels in IBCs relative to normal breast tissues with some overexpression in the TNC subtype [9], confirming some data [20]. In the present study, in order to further explore EGFR spatiotemporal regulation by the endocytic network, we selected and analyzed 3 molecules implicated in EGFR sequestration, degradation, and inhibition (i.e., MDGI, MIG6, and EIG121) in a series of 440 unilateral IBCs from patients with known clinical, pathological, and biological (including PIK3CA mutation and EGFR expression) status and long-term outcome.

MDGI (also known as FABP3) is a small $15 \mathrm{kDa}$ protein that belongs to the family of fatty acid-binding proteins (FABPs). This cytosolic molecule plays a role in differentiation of epithelial cells $[21,22]$. The MDGI gene is silenced by hypermethylation in human breast cancer cell lines and in some primary breast carcinomas, suggesting a tumor suppressor role [23]. In breast cancer cell lines, MDGI plays an important role in EGFR subcellular relocalization into an intracellular pool where the receptor is active but in a compartment that renders anti-EGFR antibody therapy inefficient [3]. MDGI interacts with integrin alpha subunits and suppresses integrin activity and cell migration and invasion [24]. In our series, we documented MDGI underexpression in IBCs $(25.7 \%$ by RT-PCR and $19.4 \%$, by IHC) and more particularly in the TNC subtype $(33.3 \%$ by RT-PCR and $27.7 \%$, by IHC). Nevertheless, we also observed mRNA MDGI overexpression in IBCs $(12.7 \%$ at the mRNA level and $13.6 \%$ at the protein level). MDGI overexpression that induces EGFR intracellular accumulation may represent a potentially new molecular mechanism of resistance when using EGFR antibodies.

MIG6 (also known as RALT) is a cytosolic protein whose centrally located ErbB-binding region (EBR) allows specific binding to members of the ErbB receptor family, including ERBB2/HER2 [25]. MIG6 gene maps on human chromosome $1 \mathrm{p} 36$, one of the regions most often targeted by genomic alterations in human tumors [26]. MIG6 suppresses EGFR receptor signalling through (i) catalytic inhibition in an inactive allosteric configuration through its EBR, (ii) receptor downregulation through RALT endocytic domain (RED) interactions with endocytic proteins AP2 and intersectins, (iii) clathrin-mediated endocytosis (CME) to late endosomes and sorting through its binding to the SNARE 


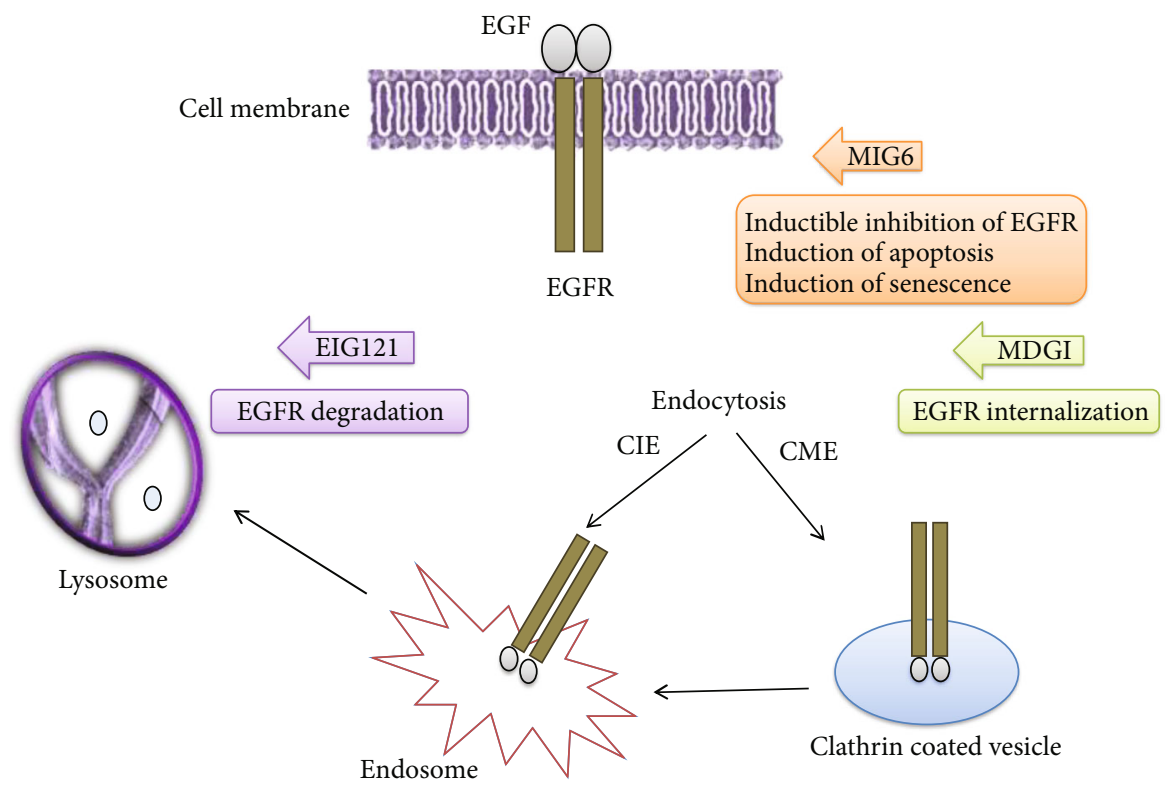

FIGURE 5: EGFR posttranslational regulators MDGI, MIG6, and EIG121 signalling pathways. EGF binding causes ligand-receptor complex to internalize via clathrin. Liganded EGFR monomers dimerize and translocate along the plasma membrane until association with a membrane domain that is enriched with clathrin on the intracellular face. This domain invaginates to initiate a clathrin-coated pit, which pinches off to form a clathrin-coated vesicle. Clathrin is shed from this primary vesicle to produce an intermediate vesicle that fuses with and delivers EGF/EGFR complex to an early endosome. The early endosome then matures into a late endosome and delivers cargo to lysosome.

protein syntaxin 8 (STX8), and (iv) downstream inhibition of EGFR signalling, including activation of ERKs and AKT as well as proliferation and cell mobility [27-37]. MIG6 has an important suppressive function since knockout mice are highly susceptible to cancer formation and MIG6 expression is lost in various cancer cells [27]. Moreover, MIG6 is one of the EGFR inducible feedback inhibitors (IFIs). IFIs have been recently identified as important regulators that reduce EGFR activity and expression at the cell surface. IFIs are actually considered tumor suppressors and gatekeepers of EGFR mitogenic signalling and antagonists of EGFR-driven tumorigenesis [34].

In mammary glands, ductal morphogenesis involves both epithelial cell autonomous, microenvironmental, and paracrine factors. Recent research on mammary gland's development in the ERRFI1 null mice showed that treatment of mammary epithelial cells with specific ErbB inhibitors AG825 (ErbB2), gefitinib (EGFR), or lapatinib (EGFR and ErbB2) prior to EGF deprivation failed to rescue the EGFindependent survival of ERRFI1 (encoding MIG6) cells [30]. MIG6 has also a p73-dependant proapoptotic role in breast morphogenesis [31]. Thus, MIG6 regulates mammary epithelial cell death independent of its role as a negative regulator of the ErbB receptors. MIG6 has also been implicated in replicative senescence, and MIG6 or pharmacologicaldriven EGFR inhibition promotes senescence of HPV-16infected cervical epithelial cells [38]. These results suggest that MIG6 could variably influence cellular homeostasis through growth inhibition, apoptosis, and senescence induction [38-41]. A recent study confirms that lung cancer stem cells inversely express EGFR and MIG6 genes [42]. In endometrial carcinoma, MIG6 plays a tumor-suppressor role by promoting epithelial cell apoptosis through the inhibition of ERK2 phosphorylation [33]. Moreover, MIG6 orchestrate epithelial-mesenchymal transition-associated kinase switch and mediate the reduction of EGFR [35].

In the present study, we found that MIG6 expression level was underexpressed with a high frequency in IBCs (45\% by RT-PCR and $43.2 \%$ by IHC). MIG6 underexpression was associated with lymph node and ER positivity. To our knowledge, this is the first description of frequencies and patterns of expression of MIG6 in a large panel of IBCs. MIG6/EGFR ratio could be tested as a new biomarker for predicting TKI response in breast carcinomas.

EIG121 was initially found overexpressed in estrogendependent endometrial cancer cell lines, leading to inhibition of cell growth and apoptosis. More recent data have identified EIG121 as a transmembrane protein associated with plasma membrane and trans-Golgi/late endosomal-lysosomal compartments enhancing lysosomal degradation of long-lived proteins [43]. Moreover, EIG121 may have important role in autophagy after nutrient deprivation and exposure to cytotoxic chemotherapeutic agents by linking EGFR with the autophagosome marker microtubule-associated protein light chain-3 (LC3). During autophagy, EIG121 and LC3 translocate into the same autophagosome vesicles and are degraded by a lysosomal mechanism. A previous mechanistic study revealed that EIG121 is located to the late endosomelysosome compartments and regulates autophagy, a cellular prosurvival mechanism activated when a cell is stressed by lack of nutrients or chemotherapy. It was shown that EIG121 conferred protection against induction of apoptosis on cells exposed to serum starvation and treatment with taxane chemotherapy [44]. In IBCs, EIG121 overexpression predicts improved survival, in agreement with the present study (Figure 1). Our results also showed high frequency of 
EIG121 underexpression in the TNC subtype $(60.3 \%$ by RTPCR and $61.1 \%$ by IHC), which has generally a poor prognosis. EIG121 underexpression was also associated with SBR histological grade III and ER and PR negativity.

Taken together, our current results on EGFR and endosomal pathway in IBCs have revealed frequent altered expressions of the three endocytic pathway regulators MDGI, EIG121, and MIG6, respectively, implicated in EGFR internalization, degradation, and inducible inhibition. These results underline the growing importance of posttranslational trafficking molecules in EGFR regulation and highlight the emerging role of EGFR IFIs as antagonists of EGFRdriven tumorigenesis. Altered expression of these three EGFR posttranslational regulators could be sufficient to deregulate inhibition and endolysosomal degradation leading to deficient termination of EGFR signalling. Moreover, implication of EIG121 in autophagy and MIG6 in apoptosis and senescence could potentially create an aberrant EGFRmediated oncogenic signalling pathway in IBCs, irrespective of EGFR levels of expression.

In conclusion, these data demonstrate a complex pattern of EGFR posttranslational regulator expression and suggest that MDGI, MIG6, and EIG121 expression statute may then represent useful biomarkers, particularly in triple-negative invasive breast carcinomas. Among these three molecules, EIG121 expression is significantly correlated with MFS and could thus represent a pertinent biomarker for EGFRtargeted therapy.

\section{Data Availability}

The data used to support the findings of this study are available from the corresponding author upon request.

\section{Conflicts of Interest}

The authors declare that they have no competing interests.

\section{Acknowledgments}

The results of this manuscript were obtained from the samples used in Dr. Didier Meseure's thesis. This work was supported by the Comité départemental des Hauts-de-Seine de la Ligue Nationale Contre le Cancer and the Association pour la recherche en cancérologie de Saint-Cloud (ARCS). We also thank the staff of Curie Institute-Rene Huguenin Hospital for their assistance in specimen collection and patient care.

\section{Supplementary Materials}

Supplementary 1. Table 1S: relationship between EGFR, MDGI, MIG6, and EIG121 gene mRNA levels in the 440 IBCs.

Supplementary 2. Table 2S: relationship between MDGI transcript levels and classical clinical biological parameters in a series of 440 breast cancer.

Supplementary 3. Table 3S: relationship between MIG6 transcript levels and classical clinical biological parameters in a series of 440 breast cancer.
Supplementary 4. Table 4S: relationship between EIG121 transcript levels and classical clinical biological parameters in a series of 440 breast cancer.

\section{References}

[1] N. Jura, N. F. Endres, K. Engel et al., "Mechanism for activation of the EGF receptor catalytic domain by the juxtamembrane segment," Cell, vol. 137, no. 7, pp. 1293-1307, 2009.

[2] T. P. Garrett, N. M. McKern, M. Lou et al., "Crystal structure of a truncated epidermal growth factor receptor extracellular domain bound to transforming growth factor alpha," Cell, vol. 110, no. 6, pp. 763-773, 2002.

[3] J. L. Macdonald and L. J. Pike, "Heterogeneity in EGF-binding affinities arises from negative cooperativity in an aggregating system," Proceedings of the National Academy of Sciences of the United States of America, vol. 105, no. 1, pp. 112-117, 2008.

[4] Z. Wang, P. A. Longo, M. K. Tarrant et al., "Mechanistic insights into the activation of oncogenic forms of EGF receptor," Nature Structural \& Molecular Biology, vol. 18, no. 12, pp. 1388-1393, 2011.

[5] D. F. Stern, "ERBB3/HER3 and ERBB2/HER2 duet in mammary development and breast cancer," Journal of Mammary Gland Biology and Neoplasia, vol. 13, no. 2, pp. 215-223, 2008.

[6] T. Mitsudomi and Y. Yatabe, "Epidermal growth factor receptor in relation to tumor development: EGFR gene and cancer," FEBS Journal, vol. 277, no. 2, pp. 301-308, 2010.

[7] I. Okamoto, "Epidermal growth factor receptor in relation to tumor development: EGFR-targeted anticancer therapy," FEBS Journal, vol. 277, no. 2, pp. 309-315, 2010.

[8] Y. Yatabe, T. Takahashi, and T. Mitsudomi, "Epidermal growth factor receptor gene amplification is acquired in association with tumor progression of EGFR-mutated lung cancer," Cancer Research, vol. 68, no. 7, pp. 2106-2111, 2008.

[9] D. Meseure, S. Vacher, K. Drak Alsibai et al., "Profiling of EGFR mRNA and protein expression in 471 breast cancers compared with 10 normal tissues: a candidate biomarker to predict EGFR inhibitor effectiveness," International Journal of Cancer, vol. 131, no. 4, pp. 1009-1010, 2012.

[10] D. C. Hyatt and B. P. Ceresa, "Cellular localization of the activated EGFR determines its effect on cell growth in MDA-MB468 cells," Experimental Cell Research, vol. 314, no. 18, pp. 3415-3425, 2008.

[11] E. Kostaras, G. Sflomos, N. M. Pedersen, H. Stenmark, T. Fotsis, and C. Murphy, "SARA and RNF11 interact with each other and ESCRT-0 core proteins and regulate degradative EGFR trafficking," Oncogene, vol. 32, no. 44, pp. 5220-5232, 2013.

[12] I. Bieche, P. Onody, I. Laurendeau et al., "Real-time reverse transcription-PCR assay for future management of ERBB2based clinical applications," Clinical Chemistry, vol. 45, no. 8 , pp. 1148-1156, 1999.

[13] I. Bieche, B. Parfait, I. Laurendeau, I. Girault, M. Vidaud, and R. Lidereau, "Quantification of estrogen receptor alpha and beta expression in sporadic breast cancer," Oncogene, vol. 20, no. 56, pp. 8109-8115, 2001.

[14] P. Chomczynski and N. Sacchi, "Single-step method of RNA isolation by acid guanidinium thiocyanate-phenol-chloroform extraction," Analytical Biochemistry, vol. 162, no. 1, pp. 156159, 1987.

[15] K. D. Awadelkarim, C. Callens, C. Rossé et al., "Quantification of PKC family genes in sporadic breast cancer by qRT-PCR: 
evidence that $\mathrm{PKC} \iota / \lambda$ overexpression is an independent prognostic factor," International Journal of Cancer, vol. 131, no. 12, pp. 2852-2862, 2012.

[16] M. Cizkova, A. Susini, S. Vacher et al., "PIK3CAmutation impact on survival in breast cancer patients and in ER $\alpha, \mathrm{PR}$ and ERBB2-based subgroups," Breast Cancer Research, vol. 14, no. 1, p. R28, 2012.

[17] D. Zardavas, L. te Marvelde, R. L. Milne et al., "Tumor PIK3CA genotype and prognosis in early-stage breast cancer: a pooled analysis of individual patient data," Journal of Clinical Oncology, vol. 36, no. 10, pp. 981-990, 2018.

[18] J. S. Rush, L. M. Quinalty, L. Engelman, D. M. Sherry, and B. P. Ceresa, "Endosomal accumulation of the activated epidermal growth factor receptor (EGFR) induces apoptosis," The Journal of Biological Chemistry, vol. 287, no. 1, pp. 712722, 2012.

[19] B. P. Ceresa, "Spatial regulation of epidermal growth factor receptor signaling by endocytosis," International Journal of Molecular Sciences, vol. 14, no. 1, pp. 72-87, 2013.

[20] D. M. Abd el-Rehim, S. E. Pinder, C. E. Paish et al., "Expression and co-expression of the members of the epidermal growth factor receptor (EGFR) family in invasive breast carcinoma," British Journal of Cancer, vol. 91, no. 8, pp. 1532-1542, 2004.

[21] H. L. Wang and A. Kurtz, "Breast cancer growth inhibition by delivery of the MDGI-derived peptide P108," Oncogene, vol. 19, no. 20, pp. 2455-2460, 2000.

[22] Y. Shen, G. Song, Y. Liu et al., "Silencing of FABP3 inhibits proliferation and promotes apoptosis in embryonic carcinoma cells," Cell Biochemistry and Biophysics, vol. 66, no. 1, pp. 139146, 2013.

[23] J. Nevo, E. Mattila, T. Pellinen et al., "Mammary-derived growth inhibitor alters traffic of EGFR and induces a novel form of cetuximab resistance," Clinical Cancer Research, vol. 15, no. 21, pp. 6570-6581, 2009.

[24] J. Nevo, A. Mai, S. Tuomi et al., "Mammary-derived growth inhibitor (MDGI) interacts with integrin $\alpha$-subunits and suppresses integrin activity and invasion," Oncogene, vol. 29, no. 49, pp. 6452-6463, 2010.

[25] L. Fiorentino, C. Pertica, M. Fiorini et al., "Inhibition of ErbB-2 mitogenic and transforming activity by RALT, a mitogeninduced signal transducer which binds to the ErbB-2 kinase domain," Molecular and Cellular Biology, vol. 20, no. 20, pp. 7735-7750, 2000.

[26] G. Ragnarsson, G. Eiriksdottir, J. T. Johannsdottir, J. G. Jonasson, V. Egilsson, and S. Ingvarsson, "Loss of heterozygosity at chromosome 1p in different solid human tumours: association with survival," British Journal of Cancer, vol. 79, no. 9-10, pp. 1468-1474, 1999.

[27] Y.-W. Zhang, B. Staal, Y. Su et al., "Evidence that MIG-6 is a tumor-suppressor gene," Oncogene, vol. 26, no. 2, pp. 269276, 2007.

[28] H. Ying, H. Zheng, K. Scott et al., "MIG6 controls EGFR trafficking and suppresses gliomagenesis," Proceedings of the National Academy of Sciences of the United States of America, vol. 107, no. 15, pp. 6912-6917, 2010.

[29] I. Ferby, M. Reschke, O. Kudlacek et al., "MIG6 is a negative regulator of EGF receptor-mediated skin morphogenesis and tumor formation," Nature Medicine, vol. 12, no. 5, pp. 568573, 2006.

[30] S. Anastasi, M. F. Baietti, Y. Frosi, S. Alema, and O. Segatto, "The evolutionarily conserved EBR module of RALT/MIG6 mediates suppression of the EGFR catalytic activity," Oncogene, vol. 26, no. 57, pp. 7833-7846, 2007.

[31] S. Hopkins, E. Linderoth, O. Hantschel et al., "Mig 6 is a sensor of EGF receptor inactivation that directly activates c-Abl to induce apoptosis during epithelial homeostasis," Developmental Cell, vol. 23, no. 3, pp. 547-559, 2012.

[32] Y. N. Wang and M. C. Hung, "Nuclear functions and subcellular trafficking mechanisms of the epidermal growth factor receptor family," Cell \& Bioscience, vol. 2, no. 1, p. 13, 2012.

[33] T. H. Kim, H. L. Franco, S. Y. Jung et al., "The synergistic effect of Mig-6 and Pten ablation on endometrial cancer development and progression," Oncogene, vol. 29, no. 26, pp. 3770 $3780,2010$.

[34] J. Kim, Y. Zhang, M. Skalski et al., "microRNA-148a is a prognostic oncomiR that targets MIG6 and BIM to regulate EGFR and apoptosis in glioblastoma," Cancer Research, vol. 74, no. 5, pp. 1541-1553, 2014.

[35] E. Izumchenko, X. Chang, C. Michailidi et al., "The TGF $\beta$ miR200-MIG6 pathway orchestrates the EMT-associated kinase switch that induces resistance to EGFR inhibitors," Cancer Research, vol. 74, no. 14, pp. 3995-4005, 2014.

[36] Y. Frosi, S. Anastasi, C. Ballarò et al., "A two-tiered mechanism of EGFR inhibition by RALT/MIG6 via kinase suppression and receptor degradation," The Journal of Cell Biology, vol. 189 , no. 3 , pp. $557-571,2010$.

[37] O. Segatto, S. Anastasi, and S. Alema, "Regulation of epidermal growth factor receptor signalling by inducible feedback inhibitors," Journal of Cell Science, vol. 124, no. 11, pp. 1785-1793, 2011.

[38] C. D. Woodworth, L. P. Diefendorf, D. F. Jette et al., "Inhibition of the epidermal growth factor receptor by erlotinib prevents immortalization of human cervical cells by human papillomavirus type 16," Virology, vol. 421, no. 1, pp. 19-27, 2011.

[39] B. Xie, L. Zhao, H. Chen, B. Jin, Z. Mao, and Z. Yao, "The mitogen-inducible gene- 6 is involved in regulation of cellular senescence in normal diploid fibroblasts," Biology of the Cell, vol. 105, no. 10, pp. 488-499, 2013.

[40] S. Anastasi, L. Castellani, S. Alemà, and O. Segatto, "A pervasive role for MIG6 in restraining cell proliferation," Cell Death and Differentiation, vol. 21, no. 3, pp. 345-347, 2014.

[41] X. Chang, E. Izumchenko, L. M. Solis et al., "The relative expression of Mig 6 and EGFR is associated with resistance to EGFR kinase inhibitors," PLoS One, vol. 8, no. 7, article e68966, 2013.

[42] Z. Xiao, B. Sperl, S. Gärtner et al., "Lung cancer stem cells and their aggressive progeny, controlled by EGFR/MIG6 inverse expression, dictate a novel NSCLC treatment approach," Oncotarget, vol. 10, no. 26, pp. 2546-2560, 2019.

[43] L. Deng, J. Feng, and R. R. Broaddus, "The novel estrogeninduced gene EIG121 regulates autophagy and promotes cell survival under stress," Cell Death \& Disease, vol. 1, no. 4, article e32, 2010.

[44] D. S. Oh, M. A. Troester, J. Usary et al., "Estrogen-regulated genes predict survival in hormone receptor positive breast cancers," Journal of Clinical Oncology, vol. 24, no. 11, pp. 16561664, 2006. 